\title{
Smart Microgrids and Virtual Power Plants in a Hierarchical Control Structure
}

\author{
T. L. Vandoorn, B. Zwaenepoel, J. D. M. De Kooning, B. Meersman and L. Vandevelde
}

\begin{abstract}
In order to achieve a coordinated integration of distributed energy resources in the electrical network, an aggregation of these resources is required. Microgrids and virtual power plants (VPPs) address this issue. Opposed to VPPs, microgrids have the functionality of islanding, for which specific control strategies have been developed. These control strategies are classified under the primary control strategies. Microgrid secondary control deals with other aspects such as resource allocation, economic optimization and voltage profile improvements. When focussing on the control-aspects of DER, VPP coordination is similar with the microgrid secondary control strategy, and thus, operates at a slower time frame as compared to the primary control and can take full advantage of the available communication provided by the overlaying smart grid. Therefore, the feasibility of the microgrid secondary control for application in VPPs is discussed in this paper. A hierarchical control structure is presented in which, firstly, smart microgrids deal with local issues in a primary and secondary control. Secondly, these microgrids are aggregated in a VPP that enables the tertiary control, forming the link with the electricity markets and dealing with issues on a larger scale.
\end{abstract}

Index Terms-microgrid, distributed energy resources, virtual power plant, primary and secondary control, droop control

\section{INTRODUCTION}

Despite the tremendous benefits of distributed energy resources (DER), some challenges arise from their penetration in the network. For instance, the capacity limits in terms of maximum power or voltage are reached more frequently and the changed, even bidirectional, power flows impact the network operation. To deal with the intermittent nature of an increasing share of DER and the increasing electricity demand, the future electrical power system will need to become more intelligent. However, the advent of the smart electrical grid will not be through a revolution, this is only possible through a gradual evolutionary change of the electrical network [1]. Because of their flexibility and scalability, microgrids have often been pointed out as a main actor in the development of the smart grid. It is expected that smart microgrids will emerge in the electrical network and the smart grid will constitute of integrated smart microgrids. These smart microgrids and separate DER can be aggregated into a virtual power plant (VPP) to behave like a conventional central generator. VPPs

This work is financially supported by the FWO-Vlaanderen (Research Foundation - Flanders, Belgium). T. Vandoorn thanks the FWO for the Fellowship received. The research of J. D. M. De Kooning is funded by the Special Research Fund (BOF) of Ghent University (Belgium). The research was carried out in the frame of the FWO Project G.0587.07N and the Inter University Attraction Poles programme IAP-VI-021, funded by the Belgian Government.

T. L. Vandoorn, J. D. M. De Kooning, B. Meersman and L. Vandevelde are with the Electrical Energy Laboratory (EELAB), Department of Electrical Energy, Systems and Automation (EESA), Ghent University, SintPietersnieuwstraat 41, B-9000 Ghent, Belgium, Phone: +32 926434 22, e-mail: Tine.Vandoorn@UGent.be

B. Zwaenepoel is with Power-Link, the energy knowledge platform of Ghent University (www.power-link.ugent.be), Wetenschapspark 1, Plassendale I, B-8400 Oostende, Belgium can contribute to the DER integration in the smart grid by fully benefiting from the two-way communication and control functionalities provided by the smart grid. According to [2], it seems likely that the best way to deal with the increasing complexity in the distribution level is a VPP or (smart) microgrid topology, or a hierarchical network structure with both topologies layered on top of each other. Both VPPs and microgrids are attractive to provide a coordinated integration of DER in the electricity network and have in common that they aggregate DER. Microgrids are an aggregation of DER that can operate both in grid-connected and islanded mode. An important benefit is that the microgrid presents itself to the electrical network as a controllable entity. VPPs form an aggregation of DER as well, but this aggregation can be virtual, thus, software-based. Hence, the geographical limits of microgrid systems are removed, but islanding of the whole VPP is often not possible. VPPs can also consist of an aggregation of microgrids as shown in Fig. 1. Hence, the concepts of smart microgrids and VPPs are similar with respect to the DER control.

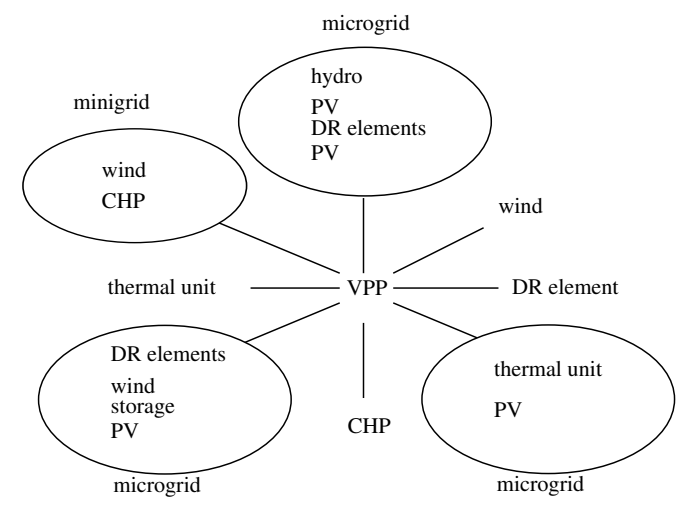

Fig. 1. Microgrids and separate DERs (DG units, demand response (DR) loads and storage elements) aggregated in a VPP: focus on the control aspects of DER

In microgrids, a distinction between primary and secondary control can be made. The microgrid primary control often avoids the usage of communication as it is responsible for a robust stable operation of the network. The primary control of the distributed generation (DG) units is different in the gridconnected mode from the islanded mode. In the grid-connected mode, the DG units are current-controlled and deliver a prespecified amount of power in the network. In the islanded mode, the DG units are voltage-controlled and are responsible for both voltage and power control of the microgrid. The primary control is responsible for the reliability and stability of the system, and thus needs a fast control strategy. The secondary control operates on a slower time frame and deals with other aspects of the system, such as the power exchange between utility network and microgrid, resource allocation, 
voltage profile optimization and frequency restoration. Hence, this control can benefit from using communication. For the active loads, again a distinction between primary and secondary demand response (DR) can be made. Like the primary DG control, the primary DR is responsible for reliability issues such as stabilizing the microgrid. It operates very fast, comparable with the time response of the DG units, and hence does not require inter-unit communication. The primary DR can be combined with the more conventional secondary DR. This operates on a slower time frame, is responsible for issues such as economic optimization and resource allocation, and is part of the smart grid concept.

From the utility perspective, the secondary control signals only need to be communicated to the PCC instead of the all smart meters in the microgrid. This delivers important scaling advantages and mitigates the communication burden.

VPPs are not capable of islanding, hence, their primary control is similar to that of the DG units in the grid-connected mode. By aggregating DER, the VPP can participate in the electricity markets, deal with congestion problems and optimize voltage profiles in its feeders. As VPPs are softwarebased, like microgrid secondary control, they benefit from the communication functionalities delivered by the smart grid changes in the network. Therefore, the feasibility of the microgrid secondary/tertiary control for VPP coordination is studied in this paper by identifying a hierarchical control in the aggregated VPP/microgrid network.

In this paper, a hierarchical structure is discussed with a VPP consisting of several microgrids. In this way, the communication and computational burden can be reduced and a more agent-based approach is taken. The VPP only communicates to the microgrid coordinator instead of to all microgrid elements.

\section{MICROGRID CONTROL}

Most DER installed in the microgrid are not suitable for direct connection to the electrical network. This is due to the characteristics of the energy produced, as many distributed generation units, such as most wind turbines, photovoltaic systems and fuel cells, do not generate a $50 / 60 \mathrm{~Hz}$ voltage. Therefore, most units use a converter for the coupling with the grid and the control of those converters has become an important concern for the grid operation.

The control objectives of smart microgrids can be divided into two categories based on the reaction time of the controllers, namely: primary and secondary/tertiary control. The primary controller generally needs to be a fully distributed controller with a very fast time response and is responsible for the stability of the system. The secondary/tertiary controllers can operate with larger time steps with limited data-exchange because of the low sampling time.

Firstly, some primary control strategies of the DG units in the microgrid are summarized. Secondly, the secondary control of both the DG units and the active loads, that overlays the primary control, is discussed.

\section{A. Primary control strategies}

The primary control objectives are different in the gridconnected mode compared to the islanded mode of the microgrid. Therefore, the grid-connected mode and islanded mode are discussed separately.

\section{1) Grid-connected mode}

In the grid-connected mode, the DG units in the microgrid deliver a predefined amount of power $P_{\text {pre }}$ to the electrical network. As the grid voltage is determined by the utility network, these modules are grid-following units. This means that calculated from $P_{\text {pre }}$ and the measured terminal voltage $V_{\mathrm{g}}$, the output current of the current-controlled units is injected.

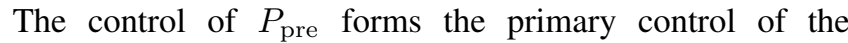
DG units and ensures their stable operation. Currently, the larger central generators are responsible for stabilizing the electrical network, thus, for the network's primary control. In a secondary control, the active power at the PCC can be controlled such that the microgrid can support the main grid. Not considered in this paper, are also power quality issues, e.g. harmonic compensation at the PCC such that the microgrid can support the power quality of the main grid.

i) Conventional strategy

Currently, $P_{\text {pre }}$ is defined by the DG units itself, e.g., by means of maximum power-point tracking in case of solar panels and wind turbines. Also, in case of CHP units, $P_{\text {pre }}$ is generally determined by the heat demand of the unit. Therefore, the output power $P_{\text {out }}$ of the unit is equal to $P_{\text {pre }}$, which is independent of the state of the electrical network.

Hence, the primary control currently follows a fit-and-forget strategy of incorporating DG into the network. The DG units, that are often based on renewable/intermittent energy sources, inject their available power into the network independent of the state of the latter. Often, a power-factor-one control is used such that the DG units inject only active power. However, the increased amount of such small generators in the distribution networks poses a significant burden on the network, for example because of voltage profile changes. In some critical times, a high input from the DG units can locally lead to over-voltage conditions. The state-of-the-art DG units are equipped with on/off control, such that over-voltage triggers the disconnection of the DG units. This leads to a significant reduction of the captured renewable energy and can pose instabilities or swings because of repetitive on and off switching of DG units.

ii) Smart grid controllers

Because of the increasing share of DG units with intermittent variable nature and where $P_{\text {pre }}$ is not determined by the state of the network, some problems may arise. Especially in the distribution network, a changed voltage profile with overvoltages in case of high renewable output and bidirectional power flows that can interact with the grid protection can cause problems in the network. Therefore, by making $P_{\text {out }}$ different from $P_{\text {pre }}$, the state of the network can be taken into account. This can be done in different ways, with and/or without interunit communication, and coordinated by a smart grid central controller (here assumed to be exploited by the distribution network operator (DNO)). When avoiding communication, the set point change of active power can be drooped with the terminal voltage of the DG unit. This can be done in a manner analogous to the voltage-based droop control strategy of islanded microgrids as discussed below. Also, the smart grid can intervene by using inter-unit communication. Both methods may lead to a higher penetration limit of renewable energy resources in the electrical network. A disadvantage of the communication approach of primary control is however the 
so-called data tsunami. The primary control deals with short time frames and a large number of high-priority messages, which increases the data burden of the system significantly. Communication-based secondary control is less exposed to these disadvantages as it operates slower and with lowerpriority messaging.

iii) Smart microgrid coordinators

The integration of DG can become smoother by combining DG units into microgrids in a coordinated manner and with active control of the output power of these units. In the primary control, the smart microgrid coordinator determines the set points of the DG units dependent on the microgrid state and information achieved from the distribution network. By aggregating groups of DG units into microgrids, the complexity of the network from a grid operators point of view can be reduced. Hereto, the DNO only coordinates the PCC power of the microgrid. Therefore, he only dispatches control signals to the microgrid coordinator instead of providing specific signals to each DG unit in the microgrid. In this way, from the DNO's point of view, the microgrid reacts as a controllable entity. The microgrid coordinator communicates with the DG units in the microgrid. Analogous to the previous approach, this is a very straight-forward method as set points are directly influenced. Although less data transfer is required in the entire network, still the data burden inside the microgrid is significant and the introduction of the smart microgrid operator can be another disadvantage of the method.

iv) Hierarchical control

The set points can also be determined locally by the DG units in a primary control, while the secondary control with smart microgrid coordinators can change these set points dependent on the microgrid needs and the DNO signals. In this way, the microgrid coordinator needs to communicate slower compared to the primary control. This is more beneficial compared to the case where the microgrid coordinator is responsible for the primary control.

Instead of a smart microgrid operator that distributes the secondary control signals, also a so-called smart transformer (ST) can be used. At the point of common coupling (PCC) of the smart microgrid to the rest of the utility network, often a transformer is in place. This transformer can be made smart in the sense that by changing its microgrid side voltage, the state of the microgrid can be altered [3]. The microgrid elements can then react on this voltage change by changing their $P_{\text {out }}$. When using the voltage-based control strategy, which is discussed further, the microgrid elements can use the same control strategy in grid-connected and islanded mode. The DNO only distributes signals to the ST, and the microgrid elements respond automatically. This reduces both the communication and computational complexity of the system. The ST concept, to which the DG units automatically respond, can also be combined with a smart microgrid operator, which requires communication with the DG units. In this way, a hierarchical control with minimal data transfer and extra redundancy and functionality can be achieved.

\section{2) Islanded mode} for:

In the islanded mode, the microgrid elements are responsible

- Voltage control:
The microgrid voltage is not determined by the utility network, but by the microgrid elements that are gridforming, and thus, voltage-controlled.

- Power balancing and power sharing:

The required active and reactive power need to be shared evenly, thus according to the ratings of the DG units.

- Power quality:

High power quality needs to be assured in the microgrid, e.g., by reactive power compensation, voltage profile regulation and harmonic current sharing inside the microgrid.

In this paper, some active power control strategies are summarized, they can be either communication-based or not dependent on communication.

\section{Communication-based control strategies}

\section{i) Central control}

In the central control method, a central controller coordinates the DG units [4]. The central controller measures the total load current and distributes this to the modules. It also distributes the voltage error to the participating modules.

ii) Master/slave control

In this control strategy, the master unit is responsible for the voltage control and distributes the reference current to the slave units [5]. The slaves are current-controlled while the master is voltage-controlled.

iii) Instantaneous (average) current sharing

In this method, the voltage and current references are shared among the modules. The references of the voltage loop are synchronized to make the output voltages of all converters in phase [6]. A current-sharing bus determines the current reference of all units using the output current measurements provided by each.

All communication-based control strategies suffer from a lower redundancy and modularity compared to the methods that do not require communication. Hence, their usage is generally restricted, e.g., to single-owner systems in smaller areas.

\section{Control strategies without communication}

In order to avoid the complexity, high costs and requirement of a highly reliable supervisory and communication system for the primary control, control strategies that are not dependent on communication are very advantageous. Especially in microgrids connecting remote converters without a communication link has an obvious advantage in terms of reliability, expandability and cost. The control strategies without communication are based on the droop-control.

i) Conventional active power/grid frequency droop control

The active power/grid frequency $(P / f)$ droop control mimics the control of the large central generators [7]. The measured active power is drooped with the nominal power to determine the reference frequency in $P / f$ droops. In the conventional network, this control strategy is based on the rotating inertia of the system. As small-scale microgrids lack this significant inertia, it is generally based on the inductive nature of the microgrid lines.

This is a well-known control strategy that enables a plug-andplay integration of DG units. However, when implemented in a low-voltage microgrid, this droop method is subjected to the predominantly resistive microgrid lines.

\section{ii) Active power/grid voltage droop control}

In low-voltage microgrids, the microgrid lines have a higher $\mathrm{R} / \mathrm{X}$ value compared to high-voltage lines. Therefore, the 
linkage between $P$ and $f$ is far less than the linkage between $P$ and the grid voltage $V$. Hence, the $P / V$ droop control is gaining interest in low-voltage microgrids [8]. To provide damping in the system and to increase the equivalent $\mathrm{R} / \mathrm{X}$, the so-called resistive virtual impedance method can be implemented in the DG units [9].

\section{iii) Voltage-based droop control}

The $P / V$ droop control takes into account the resistive nature and the lack of inertia of the microgrid. Another specific characteristic of microgrids is the high share of renewable, intermittent energy sources. To take this also into account, a variant of $P / V$ droop control, namely the voltage-based droop control has been developed [10]. This control strategy enables an optimized integration of the renewable energy resources in the microgrid by means of power change delays of the renewables compared to the controllable DG units.

The dispatchable units decrease their output power with increasing voltage and vice versa for low voltages. The variable intermittent, often renewable, units, deliver nominal power to the network in case the voltage is in the normal-voltage range. In this sense, nominal power means that the power is determined by the energy source, e.g. the optimal power point in case of photovoltaic panels, thus independent of the state of the microgrid. The normal-voltage range is a voltage-band around the nominal voltage of the microgrid, which is called the constant-power band. In case the terminal voltage exceeds this constant-power band, the power of the DG unit is changed, e.g., by including small storage elements or by abandoning the maximum power point. For this, again a $P / V$ droop is used. Generalized for the cases of all DG units, dispatchable or slightly dispatchable, in the voltage-based droop control, the width of the constant-power band is dependent of the nature of the energy source, with a large band in case of renewable sources and a small one for dispatchable DG units.

This control strategy of the generators, combined with a smart transformer (or smart back-to-back converter [11]) located at the PCC enables a microgrid islanded and gridconnected operation with the same DG control strategy. The DG units in the microgrid react on the microgrid voltage, than can be influenced by the ST. The ST increases its microgridside voltage to achieve a lower power transfer from microgrid to utility network, hence to reduce the DG output power. A lower ST voltage is induced to increase the microgrid generation. Furthermore, the DNO or VPP coordinator can impact the microgrid control by communicating with the ST solely. The microgrid elements automatically respond to ST changes.

\section{B. Secondary control}

Secondary control, which is communication-based, can be included in both the DG units and the active loads.

\section{1) DG units}

The primary control is overlayed with a secondary/tertiary control that forms the link with the smart microgrid software. The overlaying control is responsible for further microgrid optimization, such as: economic optimization, set point changes of active and reactive power to follow predefined schedules, problem management, congestion management, load response. An example of this is the set-point restoration of the primary control of the renewable energy sources. In the fast-acting primary control, the renewables change their output power in case the grid voltage exceeds the constant-power band in order to minimize the loss of renewable energy and to maintain a proper power quality in the system. For this power change, storage devices can be used. As the storage capacity is limited, the secondary control can help restoring the set point of these units, e.g. by activating the load response to change the consumption in case of extreme-voltage times. In this way, the overlaying control offers an adaptive control of the microgrid dependent on the state of the loads, DG units and storage devices.

\section{2) Active loads}

Next to the DG units, also the loads can use primary and secondary demand response. The primary demand response (DR) should enable a robust (micro)grid operation. Therefore, the communication bandwidth should be limited. When using the voltage-based droop control, communication can even be avoided as the grid voltage gives accurate information about the state of the electrical network [12]. This is mainly possible because of the power change delay of the renewables compared to the dispatchable DG units. Secondary demand response operates at a slower time frame analogous to secondary DG control. By extending the existing DR strategies with real-time information, the secondary DR is a main aspect of the smart grid concept and can gradually be introduced in the system through implementation in smart microgrids.

\section{VIRTUAL POWER PLANTS}

VPPs consist of an aggregation of DER to operate as a conventional generation system with respect to controllability and market participation. Especially, the intelligent mixedasset VPPs are promising as they deliver scaling benefits and enclose different types of resources. By aggregation into VPPs, small stand-alone DER that are generally under the capacity threshold to enter the electricity market [13] can participate in the markets. Also, as many DER are based on intermittent energy sources, they face power unbalance penalties that can be mitigated by a mixed-asset VPP.

VPPs are very similar to microgrids as shown in Fig. 2 The main difference is that the microgrid units are physically located in each others proximity and have a PCC with PCC switch for the connection of the microgrid to the utility network that enables microgrids to island if necessary. The VPP units on the other hand can be located physically apart and hence, are always grid-tied. In this sense, a VPP is software-based while a microgrid depends primarily on hardware components.

An important question is how to control a VPP and whether the developed control strategies used for microgrids are applicable, probably in a changed form, in VPPs.

\section{A. Applications}

1) Contribution to congestion management: DER are now installed in the electric power system without coordination and based on a fit-and-forget strategy. Because the network operators can not yet change the output of the DER, they limit the installed DER capacity based on the worst-case scenario:

$$
\sum P_{\mathrm{max}, \mathrm{DER}}-\sum P_{\mathrm{min}, \mathrm{load}} \leq \sum P_{\mathrm{max}, \mathrm{grid}} \text {. }
$$




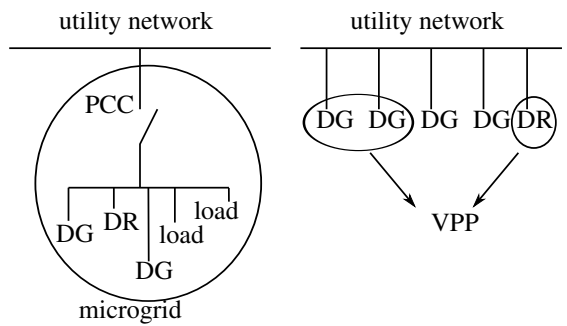

Fig. 2. Microgrids versus VPP: physical versus software-based aggregation of DER

Although this grid limit is only violated a few hours per year, the maximum installed capacity is limited permanently. As such, the maximum installed renewable energy capacity is limited. By installing a VPP in a certain region, which requires a limited investment, the DNO and transmission network operator can limit the DER capacity when necessary. As such, the real capacity factors and coincidence factors can be taken into account instead of assuming them as one in the conservative approach used in passively operated grids. This can prevent huge investments of both time and money in grid reinforcement and allows a much larger share of renewable sources to be installed.

2) Contribution to voltage profile regulation: Voltage profile regulation can be done in a hierarchical control structure. In the primary control of the DG units, conventionally, overvoltage shut down is included. In the future, by means of a droop mechanism, which can be adopted analogous to the voltage-based droop control in islanded microgrids, voltage profile optimization can be obtained by gradually changing the DG output power dependent on the terminal rms voltage. Again, power change delay of the renewables is crucial.

In a secondary control, the VPP can coordinate the DG units in order to achieve an optimized voltage profile regulation.

3) Contribution to oscillation clearance: The classical on/off control of DG, where the DG units switch off in case of over-voltage and turn on again when the voltage has decreased, is demonstrated in Fig. 3. As shown, this can lead to oscillations in the network. This primary safety function of the sources leads to unpredictable behavior. The first unit that reaches its voltage-limit will shut off. Due to tolerances in the components, it is hard to predict which unit this will be. This leads to low production rates on the days with the largest potential of renewable energy (e.g., wind or solar power). The VPP can solve this issue by providing coordination of the power changes. The on/off control can be changed to, for example, the voltage-based droop control as mentioned above. In this way, the DG output power does not change with $100 \%$, but with a value dependent on the terminal voltage of the units. This prevents voltage spikes on the grid and hence also protects the connected equipment. Also, the power decreases due to over-voltages can be shared more evenly between the available resources, gaining more (predictable) production for the system as a whole.

\section{B. Control strategies}

The main goal of aggregating DER into VPPs is to enable them to contribute in the markets and act as a conventional generator. Therefore, the VPP coordinator is responsible for

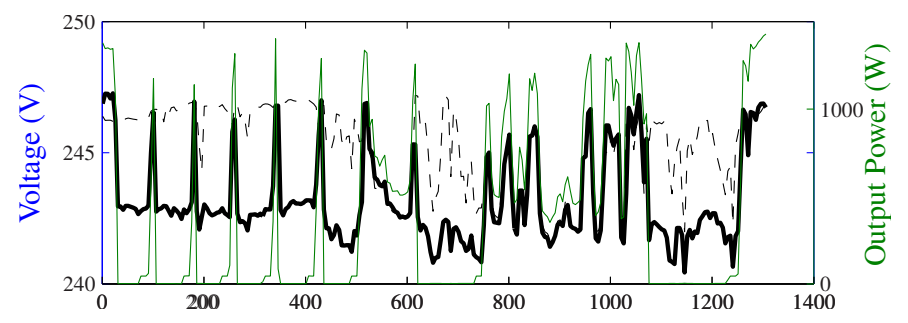

Fig. 3. On/off control of DG leading to grid-oscillations: measurements of $\mathrm{PV}$ panels in Oostende, Belgium ( $-=\mathrm{DG}$ unit $1,---=\mathrm{DG}$ unit $2, \overline{=}=$ $\left.V_{\mathrm{PCC}}\right)$

supervision, balancing control, ancillary services and market interface. This paper focusses on active power set point changes of the DG units triggered by the VPP coordinator. This set point change is slow compared to the primary control of the network, and hence is included in the secondary/tertiary grid control. In this way, the primary control strategy is not influenced by the VPP and the VPP coordinator can take advantage from the communication opportunities brought by the smart grid concept.

The DER in the VPPs can, in turn, also be aggregated into microgrids. In this way, microgrids and VPPs are layered on top of each other. The VPP influences the local controllers by distributing power set point changes to:

- the DERs separately;

- the microgrids .

For the VPP coordinator, microgrids offer the advantage of presenting themselves as entities, which reduces the communication and computational burden of the VPP coordinator. The VPP coordinator only adjusts the power through the PCC, while inside the microgrid, this power change can be dealt with taking into account all details of the microgrid in order to obtain an optimal operation.

The VPP coordinator calculates a total desired output power change of the VPP from information received from the DNO or from the VPP elements, e.g., for the balancing of the VPP. In order to realize this power change, two strategies are possible. In the direct control, the VPP coordinator determines a separate power change for each DER/microgrid and distributes this information to all the DER/microgrids in the system. The VPP coordinator has, thus, a direct control of its portfolio of DER and the performance of the system is highly linked with the intelligence of the VPP coordinator. Ideally, an optimal operation can be achieved when the two-way communication is fully exploited, but this can lead to problems of scalability, adaptability and computational burden. In case of a marketbased control, the VPP coordinator uses a VPP market to send price signals to the DER or approves DER bids [14]. Opposed to the direct control strategy, the decisions for power changes are made by the DER units locally in order to maximize their profit. The VPP coordinator can not directly control the DER but has some controllability by changing the incentives. Both methods require two-way communication, intelligence and computational burden. Here, only the control method is studied, so active power changes are defined as such, but can result from pricing or bidding strategies as well.

VPPs can be categorized in centralized VPPs or decentralized (hierarchical) VPPs. In centralized VPPs, the VPP 
dispatches the operating point of each DER. This requires that the VPP has accurate knowledge of the state of the DER. Decentralized VPPs on the other hand operate with a hierarchical architecture. In these VPPs, distributed local controllers are overlayed with either a central controller or with information exchange agents to form an integrated system. An example of a decentralized VPP is a market-based VPP model operated by a bidding or price signal scenario [15].

Next to centralized and decentralized VPP control, the control can also be based on islanded microgrid control in the case of VPPs consisting of aggregated microgrids :

- Central control (centralized control):

The VPP dispatches the control signals to the microgrid central controller. In turn, this central controller distributes power set point changes to the DER in the microgrid, which is analogous to the microgrid central control scheme. Opposed to centralized VPP control in which the VPP coordinator communicates with each DER, here, a microgrid central controller is included as extra intelligence level between the VPP and the DER.

- Master/slave control (centralized control):

The VPP dispatches the control signals to the microgrid master DER. The microgrid master coordinates the set point changes of the slave units.

- Smart transformer (or back-to-back converter control) (decentralized control in the microgrid ):

The VPP coordinator changes the ST set-points. In case of voltage-based droop control in the microgrid, the microgrid elements respond automatically to match the ST set point changes.

Communication is required:

- from the VPP to the microgrid coordinator and separate DER because the VPP elements are not physically located near each other

- inside the microgrid or from the VPP to the ST.

\section{Primary and secondary control of VPPs}

The primary control is not determined by the VPP coordinator, hence, analogous to primary control in microgrids, it is determined by the DER locally. The output power of the DG units can be altered according to the state of the network by means of (modified) $P / f, P / V$ and voltagebased droop controllers to enable the VPP units to react on local voltage/frequency changes for local power quality improvements and a stable VPP operation.

This primary control can be overlayed with a secondary control scheme to obtain an optimal operation inside the VPP. For example, the VPP coordinator can undo a primary $P_{\text {out }}$ change of one unit by changing $P_{\text {out }}$ of another unit, e.g., to reach an economic optimum.

In the tertiary control strategy, with a slower time frame, the VPP coordinator communicates with the DNO to change the power of the DG units in the VPP to reach an optimal operation on the utility-scale. In this tertiary control, the VPP coordinator forms the link between the DER and the electricity markets.

The tertiary control delivers set points for the secondary control strategy, that in turn changes the nominal power in the primary control strategy.

\section{HIERARCHICAL VPP/MICROGRID CONTROL}

Based on the primary and secondary control strategies of microgrids and the VPP control, as discussed above, a hierarchical VPP/microgrid control strategy is presented in this paper.

The primary control is responsible for a stable network operation and ideally operates based on local measurements only. This primary control is equal in grid-connected microgrids and VPPs. The DER typically lean on the conventional large generators for the primary control. However, in the future, with more and more DER, these units will also need to contribute to the networks primary control. This can be done by including $P / f$ droop controllers analogous to these in the conventional network such that these units can contribute to the frequency control of the electrical network. Another example is the usage of the voltage-based droop control like presented for the case of the islanded resistive microgrids. This method is especially interesting for dealing with local problems of the low-voltage networks that the considered DER are linked to. In this control strategy, the DER respond to local voltage changes in a delayed manner if they consist of renewable energy sources. In this way, a better power quality can be achieved and the penetration limit of renewables can be extended.

In the conventional grid control, secondary and tertiary control are described as follows [16]:

- secondary control is a centralised automatic control that delivers reserve power in order to bring back the frequency and the interchange programs to their target values;

- tertiary control is manual change in the dispatching and unit commitment in order to restore the secondary control reserve, to manage congestions, and to bring back the frequency and the interchange programs to their target if the secondary control reserve is not sufficient.

If applied to VPPs, the secondary control is a control for optimization inside the microgrid (analogous to the microgrid secondary control).

The tertiary control is enabled by the control signals sent from the VPP to the microgrids or the separate DG units. A higher level of tertiary control deals with control signals from the DNO to the VPPs or directly to the microgrids/DG units in case they are not part of a VPP.

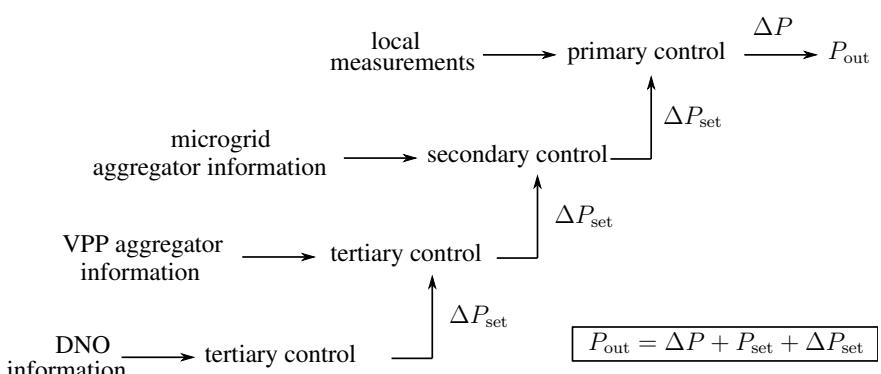

Fig. 4. Hierarchical control in VPP with microgrids

By combining these different levels of controllers, a hierarchical control is formed where each control level delivers control signals to the lower levels as represented in Fig. 4. In this figure, the hierarchical control in the network with a VPP 
consisting of multiple microgrids is summarized. In case of a VPP with separate DER units, the secondary VPP control can be omitted. In a microgrid without overlaying VPP, the VPP tertiary control is omitted. In an islanded microgrid, there is no tertiary control as defined in Fig. 4. In the state-of-theart network, the renewable DG units have no secondary and tertiary control: $P_{\text {out }}=P_{\text {set }}$, with $P_{\text {set }}$ merely determined by the DER, not by the state of the network.

\section{CONCLUSIONS}

In this paper, some microgrid control methods are summarized for both grid-connected and islanded mode. A distinction between primary and secondary control is made. Next, the feasibility of these microgrid controllers in VPPs is discussed. A hierarchical control of smart microgrids and VPPs layered on top of each other is presented. In this hierarchical control, the microgrid deals with local issues while the VPP coordinator forms the link with the electricity markets and deals with issues on the level of the distribution network (DNO).

\section{REFERENCES}

[1] H. Farhangi, "The path of the smart grid," in IEEE Power \& Energy Magazine, Jan./Feb. 2010, vol. 8, no. 1, pp. 18-28.

[2] P. Asmus, "Microgrids, virtual power plants and our distributed energy future," The Electricity Journal, vol. 23, no. 10, pp. 72-82, Dec. 2010.

[3] T. Vandoorn, J. D. Kooning, B. Meersman, J. Guerrero, and L. Vandevelde, "Voltage-based control of a smart transformer in a microgrid," IEEE Trans. Ind. Electron., 2011, accepted for publication, Digital Object Identifier: 10.1109/TIE.2011.2165463.

[4] T. F. Wu, K. Siri, and J. Banda, "The central-limit control and impact of cable resistance in currentdistribution for parallel-connected DCDC converters," in 25th Annual IEEE Power Electronics Specialists Conference (PESC '94), Taipei, Taiwan, Jun. 20-25 1994, pp. 694-702.

[5] K. Siri, C. Q. Lee, and T. F. Wu, "Current distribution control for parallel connected converters part i," IEEE Trans. Aerosp. Electron. Syst., vol. 28 , no. 3, pp. 829-840, July 1992.

[6] X. Sun, Y.-S. Lee, and D. Xu, "Modeling, analysis, and implementation of parallel multi-inverter systems with instantaneous average-currentsharing scheme," IEEE Trans. Power Electron., vol. 18, no. 3, pp. 844 856, May 2003.

[7] R. H. Lasseter and P. Paigi, "Microgrid: A conceptual solution," in Proc. IEEE Power Electron. Spec. Conf. (PESC 2004), Aachen, Germany, 2004.

[8] H. Laaksonen, P. Saari, and R. Komulainen, "Voltage and frequency control of inverter based weak LV network microgrid," in 2005 International Conference on Future Power Systems, Amsterdam, Nov. 18 2005.

[9] J. M. Guerrero, J. Matas, L. García de Vicuña, M. Castilla, and J. Miret, "Decentralized control for parallel operation of distributed generation inverters using resistive output impedance," IEEE Trans. Ind. Electron., vol. 54, no. 2, pp. 994-1004, Apr. 2007.

[10] T. L. Vandoorn, B. Meersman, L. Degroote, B. Renders, and L. Vandevelde, "A control strategy for islanded microgrids with dc-link voltage control," IEEE Trans. Power Del., vol. 26, no. 2, pp. 703-713, Apr. 2011.

[11] R. Majumder, M. Dewadasa, A. Ghosh, G. Ledwich, and F. Zare, "Control and protection of a microgrid connected to utility through backto-back converters," Electr. Power Syst. Res., vol. 81, no. 7, pp. $1424-$ $1435,2011$.

[12] T. L. Vandoorn, B. Renders, L. Degroote, B. Meersman, and L. Vandevelde, "Active load control in islanded microgrids based on the grid voltage," IEEE Trans. on Smart Grid, vol. 2, no. 1, pp. 139-151, Mar. 2011.

[13] S. Ropenus and K. Skytte, "Regulatory review and barriers for the electricity supply system for distributed generation in eu-15," Internat. Journal of distributed energy resources, vol. 3, pp. 243-257, 2007.

[14] S. You, C. Træholt, and B. Poulsen, "Generic virtual power plant: management of distributed energy resources under liberalized electricity market," in Internat. Conf. on Advances in Power System Control, Operation and Management (APSCOM 2009), Hong Kong, China, Nov. 8-11, 2009, pp. 1-6.
[15] _ , "A market-based virtual power plant," in Internat. Conf. on Clean Electrical Power (ICCEP2009), Capri, Italy, Jun. 9-11, 2009, pp. 460465.

[16] UCTE. (2009, Mar. 19,) Operation handbook policy 1: Load-frequency control and performance. [Online]. Available: http://www.entsoe.eu

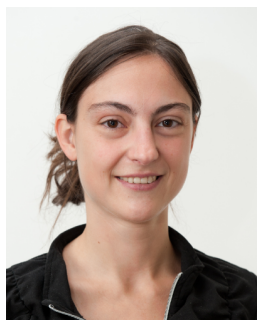

Tine Vandoorn (S'09) was born in Torhout, Belgium in 1985. She received the M.S. in electromechanical engineering (EELAB) from Ghent University, Ghent, Belgium, in 2008. In 2008, she joined the Electrical Energy Laboratory of Ghent University where she is currently pursuing the Ph.D. degree. Her present research interests include electric power systems, voltage and power control of DG units, management of (smart) microgrids. In 2009, she was awarded a grant as Ph.D. fellow of the Research Foundation - Flanders (FWO)

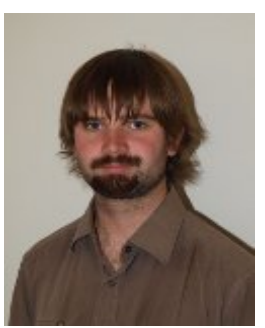

Brecht Zwaenepoel is born in Brugge, Belgium in 1987. From 2005, he studied industrial engineering at the Catholic University College of Bruges-Ostend. In 2009, he received his MSc electro-technical engineering in automation and in 2010, his MSc electrotechnical engineering in renewable energy. In Sep. 2010, he joined Power-Link, the energy knowledge platform of Ghent University. In 2011, he started his $\mathrm{Ph} . \mathrm{D}$. research regarding congestion management on high voltage lines by actively controlling decentral production units.

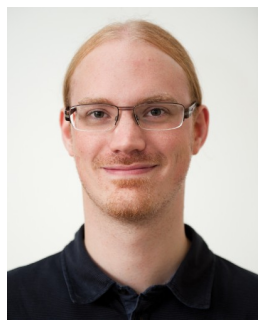

Jeroen De Kooning (S'09) was born in Kapellen, Belgium in 1987. He received the M.S. degree in electromechanical engineering from Ghent University, Belgium, in 2010. Since then, he is with the Electrical Energy Laboratory (EELAB) of Ghent University and is currently pursuing the $\mathrm{Ph}$.D degree. His present research interests include wind energy systems, control of power electronic converters and brushless ac drives.

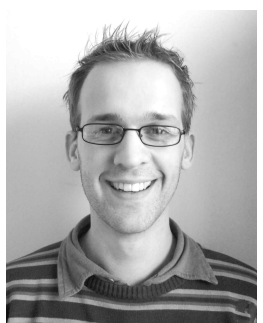

Bart Meersman (S'07) was born in Sint-Niklaas, Belgium on July 29, 1983. He received the M.S. degree in electromechanical engineering from Ghent University, Belgium, in 2006. Since then, he is with the Electrical Energy Laboratory (EELAB) of Ghent University and is currently working towards a $\mathrm{Ph} . \mathrm{D}$. degree. His present research interests include dynamic phasors, renewable energy applications, digital control of power electronic converters and their contribution to power quality.

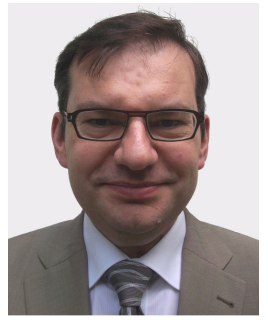

Lieven Vandevelde (M05 - SM07) was born in Eeklo, Belgium, in 1968. He graduated as Master of Electromechanical Engineering and received the $\mathrm{Ph} . \mathrm{D}$. degree from Ghent University, Belgium, in 1992 and 1997, respectively. He is with the Electrical Energy Laboratory (EELAB), Ghent University, where he has been a Professor in electrical power engineering since 2004. His research and teaching activities are in the field of electric power systems, electrical machines and (computational) electromagnetics. 